\section{Auswirkungen der Radiotherapie auf die Lunge}

\author{
Die postoperative Radiotherapie (RT) nimmt im Gesamtkonzept der \\ Brustkrebsbehandlung nach brusterhaltender Operation sowie nach \\ Mastektomie einen festen Platz ein. Wie wirkt sich das auf die Lunge aus?
}

Tram rotz der Erfolge in der Begrenzung des bestrahlten Volumens, etwa durch Akzelerierungs- und Fraktionierungstechniken, bleibt die kardiale und pulmonale Toxizität ein limitierender Faktor. Die strahleninduzierte akute Pneumonitis wurde in mehreren Studien thematisiert, eher selten hingegen Spätfolgen wie interstitielle fibrotische Veränderungen im Lungengewebe. Jetzt gibt es prospektive Daten zu den langfristigen Veränderungen der Lungenfunktion.

An der Studie nahmen 43 Patientinnen im Durchschnittsalter von 55 Jahren teil, für die eine postoperative RT indiziert war. Sie erhielten $50 \mathrm{~Gy}$ in $2 \mathrm{~Gy}$ Fraktionen; vor sowie sechs, zwölf, 24 und 84 Monate nach der Radiotherapie erfolgten Lungenfunktionstests (PFT) und eine Ventilations-/Perfusionsszintigrafie. Die Daten 41 Patientinnen gingen in die Endauswertung ein.

Die durchschnittliche inzidentielle pulmonale Strahlendosis lag bei 10,9 Gy, bei zusätzlicher Lymphknotenbestrahlung etwas höher. Sechs Monate nach initialer RT kam es zu einem Abfall aller PFT-Werte. FVC und FEV-1 stiegen dar- aufhin wieder an und erreichten bzw. übertrafen nach sieben Jahren die Ausgangswerte. Die Parameter aus den Lungen-Volumenmessungen und der V/QSzintigrafie fielen über zwei Jahre kontinuierlich und erreichten nach sieben Jahren wieder nahezu ein Normalniveau.

Fazit: Nach inzidentieller Lungenbestrahlung bei der postoperativen Radiotherapie von Brustkrebspatientinnen kommt es binnen 24 Monaten zu einem Abfall bestimmter Lungenfunktionsparameter. Diese erreichen aber nach sieben Jahren wieder ihren Ausgangswert. Der temporäre Rückgang der PFT-Werte fiel gering aus. Wolfgang Zimmermann

Jaén J et al. Long-term changes in pulmonary function after lung irradiation for breast cancer: a prospective study with 7-year follow-up. Int J Radiat Oncol Biol Phys. 2012;84(5):e565-70.

\title{
Mammakarzinom: Triple-negativ im höheren Alter
}

Der besonders aggressive triple-negative Brustkrebs (TNBC) gilt als Subtyp, den vor allem jüngere Patientinnen aufweisen. Doch er trifft häufig auch ältere Frauen. Daher lohnt sich ein Vergleich der Tumorcharakteristika.

A uch wenn der TNBC-Subtyp bei jüngeren Patientinnen besonders häufig ist: Seltener wird er erst ab der achten Lebensdekade. Zahlreiche 65-70-jährige Frauen erkranken sehr wohl an diesem aggressiven Tumorsubtyp, der sich durch

das Fehlen sowohl von Östrogen- und Progesteronrezeptoren als auch HER2Überexpression auszeichnet. Daten zu älteren Patientinnen fehlen bis heute, österreichische Kollegen analysierten retrospektiv klinisch-pathologische Cha-

Tumorcharakteristika jüngerer und älterer Patientinnen mit TNBC

Tabelle 1

Variable
Histologie
duktal
andere
Pathologische Tumorgröße
$\leq 2 \mathrm{~cm}$
$>2$ bis $\leq 5 \mathrm{~cm}$
$>5 \mathrm{~cm}$
unbekannt
Lymphknoten
Keine
$1-3$
$4-9$
$>10$
unbekannt
Trumorgrad
G1
G2
G3

TNBC-Patientinnen $<65$ Jahre $(n=192 ; 75,6 \%)$

$173(90,1 \%)$ $19(9,9 \%)$

$92(47,9 \%)$

$69(35,9 \%)$

$21(10,9 \%)$

$10(5,3 \%)$

$126(65,5 \%)$

$32(16,7 \%)$

$14(7,3 \%)$

$16(8,3 \%)$

$4(2,1 \%)$

$7(3,6 \%)$

$23(12 \%)$

$162(84,4 \%)$
TNBC-Patientinnen $\geq 65$ Jahre $(n=62,24 ; 4 \%)$

$55(88,7 \%)$

$7(11,3 \%)$

$34(54,8 \%)$

$21(33,9 \%)$

$4(6,5 \%)$

$3(4,8 \%)$

$33(53,2 \%)$

$12(19,4 \%)$

$6(9,7 \%)$

$2(3,2 \%)$

$9(14,5 \%)$

$4(6,5 \%)$

$13(21 \%)$

$45(72,6 \%)$ rakteristika von 254 TNBC-Patientinnen, die ihre Diagnose zwischen 1998 und 2004 erhielten. Verglichen wurden Tumorcharakteristika zu Beginn der Behandlung, Demografie und Muster der Metastasierung zwischen jüngeren $(<65$ Jahre; $75,6 \%)$ und älteren Patientinnen ( $\geq 65$ Jahre; $24,4 \%$ ).

Tumorgröße und -grad und die Zahl der positiven Lymphknoten unterschieden sich nicht signifikant zwischen den Gruppen. Viszerale Metastasen waren in beiden Gruppen signifikant häufiger als Knochenmetastasen $(p<0,001)$, Lokalrezidive, Knochen- und sekundäre Lymphknotenmetastasen jedoch häufiger bei jüngeren Frauen ( $p=0,035,0,025$ bzw. 0,041). Ältere Patientinnen erhielten seltener Chemotherapie $(\mathrm{p}<0,001)$.

Fazit: Der TNBC älterer Frauen sollte ebenso viel Aufmerksamkeit erhalten wie bei jüngeren Frauen. Eine Chemotherapie sollte unabhängig vom Alter erfolgen. Schließlich haben 65 -jährige $\mathrm{Pa}$ tientinnen ohne Komorbiditäten noch eine Lebenserwartung von im Mittel 20 Jahren.

Christina Berndt

Königsberg $R$ et al. Tumor characteristics and recurrence patterns in triple negative breast cancer: A comparison between younger $(<65)$ and elderly $(\geq 65)$ patients. Eur J Cancer. 2012;48(16):2962-8. 